\title{
La Educación especial en Chile, en la perspectiva del Dr. Jaime Caiceo Escudero
}

\author{
Special education in Chile, in the perspective of \\ Jaime Caiceo Escudero
}

A Educação especial no Chile, na perspectiva de Jaime Caiceo Escudero

\author{
Estela Socías Muñoz \\ Universidad Mayor (Chile) \\ https://orcid.org/0000-0003-0228-3430 \\ estela.socias@mayor.cl
}

\section{Resumen}

Basado en las investigaciones realizadas por el Dr. en Ciencias de la Educación, Don Jaime Caiceo Escudero, en relación a la educación especial, que en Chile se le denomina educación diferencial desde 1968 por el Ministerio de Educación, se pretende describir desde sus orígenes hasta la década del ochenta del siglo XX, período en el cual hubo un avance notorio de este tipo de educación en Chile. Se formulará una definición sobre la misma, recurriendo a publicaciones de Dr. Caiceo estudioso de esta área educativa, donde él realiza un análisis sobre el desarrollo histórico de este tipo de educación, desde sus inicios en el año 1852 hasta 1980, dividiéndola en tres grandes períodos, los cuales se describirán y se podrá visualizar la consolidación y las realizaciones científicas (1964-1980), indicando diversas publicaciones, como las del Dr. Luis Bravo Valdivieso. Se indicará, además, la actuación importante que ha tenido después del período estudiado de la Premio Nacional en Educación, Mabel Condemarín; ambas personalidades chilenas son reconocidas a nivel internacional.

Palabras Claves: Educación especial en Chile, educación diferencial, historia de la educación. 


\begin{abstract}
Based on research conducted by Dr. in Education Sciences, Don Jaime Caiceo Escudero, in relation to special education, which in Chile is called the education differential since 1968 by the Ministry of Education, intends to describe from their origins until the Decade of the eighties of the 20th century, period in which there was a breakthrough that is notorious for this type of education in Chile. A definition on it, will be formulated by Dr. Caiceo scholar of this educational area publications, where he carried out an analysis of the history of this type of education development, since its inception in 1852 until 1980, dividing it into three large periods, which will be described and you can view the consolidation and the scientific achievements (1964-1980), indicating different publications, such as the of the Dr. Luis Bravo Valdivieso. In addition, indicate the important action that has taken after the study period of the National Award in Education, Mabel Condemarín; both Chilean personalities are recognized at the international level.
\end{abstract}

Keywords: Special education in Chile, differential education, history of education.

\title{
RESUMO
}

Baseado nas investigações realizadas pelo Doutor em Ciências da Educação, Dom Jaime Caiceo Escudero, em relação à educação especial, que no Chile denomina-se educação diferencial desde 1968 pelo Ministério da Educação, pretende-se descrever desde suas origens até a década de oitenta do século XX, período em que houve um avanço notório deste tipo de educação no Chile. Será formulada uma definição sobre a mesma, recorrendo a publicações do Dr Caiceo, estudioso desta área educativa onde ele realiza uma análise sobre o desenvolvimento histórico deste tipo de educação, desde seu início no ano de 1852 até 1980, dividindo-a em três grandes períodos os quais se descreverão e poderá se visualizar a consolidação e as realizações científicas (1964-1980), indicando diversas publicações, como as do Dr Luis Bravo Valdivieso. Será indicado, também, a atuação importante que se tem tido depois do período estudado do Prêmio Nacional em educação, Mabel Condemarín; ambas personalidades chilenas são reconhecidas a nível internacional.

Palavras Chaves: Educação especial no Chile, Educação Diferencial, História da educação. 


\section{Introducción}

Según las investigaciones del Dr. Jaime Caiceo Escudero, recién en nuestro país entre las décadas del setenta y del ochenta del siglo pasado, la educación especial tuvo un auge notorio en Chile ya que desde esa época se han ido realizando distintas investigaciones en el área, sobre todo en los trastornos del aprendizaje. Por esto también, han surgido diversas escuelas especiales y la educación superior se ha preocupado de formar especialistas en este tipo de educación. Sin embargo, ella tiene más de un siglo y medio de historia.

En este trabajo se describe y analiza la importancia de la época en estudio, en la que hubo participación de connotadas personalidades, tanto chilenas como extranjeras, que darán inicio a un desarrollo educacional en sus diferentes ámbitos y niveles. Se demostrará la importancia que tiene la educación especial ya que con ella las personas con discapacidades, empiezan a ser reconocidas por la sociedad, buscando estrategias para impulsar este tipo de enseñanza y lograr formar maestros que puedan especializarse profesionalmente en esta área, a fin de que puedan entregar conocimientos, pero especialmente alcanzar en los alumnos con discapacidades una mejor calidad de vida; últimamente en el país ha habido reformas educativas que han impulsado la integración y la inclusión -creando el Programa de Integración Escolar-, distinguiendo a niños con Necesidades Educativas Especiales Transitorias o Permanentes, todo lo cual han significado avances notorios.

Se puede visualizar en esta investigación el desarrollo de la escuela activa, especialmente en las personas de María Montessori y Oviedo Decroly, subrayando sus aportes a la educación especial en Chile. Se demostrará el avance de este tipo de educación, analizando el pensamiento del Doctor Caiceo referente al tema expuesto y a su avance.

Este trabajo es fruto de una investigación realizada en la Universidad Mayor y la metodología es la propia de los estudios históricos, recurriendo a fuentes primarias y secundarias (artículos publicados en revistas y entrevista al investigador Jaime Caiceo Escudero) (CAICEO, 1988a; 1988b; 2005; 2008; 2009; 2010a; 2010b; 2011, 2018).

\section{Discapacidad. Definiciones entorno al concepto}

Existen varias definiciones de educación especial, y para entender un poco más es necesario precisar el concepto según la Organización de las Naciones Unidas para la Educación, la Ciencia y la Cultura (Unesco): "La enseñanza destinada a los niños y jóvenes que tienen dificultades para aprender, porque sufren cualquier deficiencia (ciegos, ambliopes, sordos, retardados mentales, alumnos lentos, con déficit fisico) o varias deficiencias combinadas" (MINISTERIO DE EDUCACIÓN DE CHILE, 1975, p. 20 en CAICEO, 2010a, pp. 32-33). En 1975, el Ministerio de Educación de Chile indicó que este tipo de educación:

Constituye un sistema de elementos técnico-administrativos, financieros, materiales y humanos, destinados a la formación integral como persona humana, de aquellos sujetos que debido a algún déficit global o específico, sociocultural, sensorial o motor, no pueden lograr su desarrollo armónico y completo a través del sistema de educación general común (MINISTERIO DE EDUCACIÓN DE CHILE, 1975, p. 19 en CAICEO, 2010a, p. 33).

El mismo Ministerio de Educación señala que según la Sociedad Internacional para la Rehabilitación de los Discapacitados, existen seis diferentes tipos de sujetos que atiende la educación especial. Ellos son: 
1. Déficit mentales: Corresponden a personas con déficit intelectual en niveles entrenables (déficit moderado o leve); 2. Deficientes sensoriales: seres humanos con déficit auditivo (sordo hipoacústico) o visual (ciego, ambliope); 3. Deficientes motores: Sujetos con alteraciones motoras del sistema nervioso central (paralítico cerebral) o minusvalía física (lisiado); 4. Personas con trastornos del lenguaje oral: Personas que padecen alteraciones centrales del lenguaje (disartrias, afasias y otras) o periféricas (dislalias, rinolalias y otras); 5. Sujetos con trastornos específicos del aprendizaje: Los que están referidos al aprendizaje de lectura y el cálculo (dislexia, disortografía, disgrafía, discalculia); 6. Algunos casos de irregulares sociales: Son los que tienen alteraciones conductuales de diversas etiologías (MINISTERIO DE EDUCACIÓN DE CHILE, 1975, p. 25 en CAICEO, 2010a, p. 33).

Para lograr comprender los significados que han existido históricamente hacia las personas con discapacidad se precisan algunos conceptos al respecto.

Según la Clasificación Internacional de Deficiencias, Discapacidades y Minusvalías (CIDDM) en el año 1980, aparece definida discapacidad como "toda restricción o ausencia (debida a una deficiencia) de la capacidad de realizar una actividad en la forma o dentro del margen que se considera normal para un ser humano. (EGEA y SARABIA, 2001 en CSASZAR, ORTEGA y RODRÍGUEZ, 2013, p. 49).

Más adelante, en el 2001, surge el Clasificador Internacional del Funcionamiento de la Discapacidad y Salud (CIF), que tiene por objetivo "Proporcionar un lenguaje unificado y estandarizado que sirva como punto de referencia para la descripción de la salud y los estados relacionados con la salud" (OMS en EGEA y SARABIA, 2001 en CSASZAR, ORTEGA y RODRÍGUEZ, 2013, p. 49).

Entre las definiciones mencionadas por los clasificadores antes mencionados, han transcurrido más de dos décadas, por lo que se puede descubrir que no hubo un avance significativo en relación a la significación y comprensión de la discapacidad, ya que aún cuando se escucha mencionar en reiteradas ocasiones los conceptos de inclusión, modelos sociales de comprensión de la discapacidad, no existe una contextualización clara ni cambios en el accionar de la sociedad hacia las personas con discapacidad.

En Chile, a través de la Ley 19.284 (1994) dedicada a la Plena Integración Social de Personas con Discapacidad, se crea el Fondo Nacional de la Discapacidad -FONADIS-, a fin de aportar recursos para apoyar a las personas con discapacidad en el país. Por otro lado, en el año 2006 el concepto de discapacidad fue comprendido explícitamente por el Fondo Nacional de la Discapacidad, considerando las definiciones otorgadas, tanto por la Organización Mundial de Salud como por el autor Miguel Ángel Verdugo. La OMS, en los 90', definió la discapacidad como: "... resultante de la interacción de una deficiencia de una persona y las variables ambientales que incluyen el ambiente fisico, las situaciones sociales y los recursos" (ZONDEK et al., 2006, p.11 en CSASZAR, ORTEGA y RODRÍGUEZ, 2013, p. 50).

Según FONADIS, existen tres tipos de discapacidad: leve, moderada y severa. Por un lado, la discapacidad leve consiste en "aquellas personas que presentan alguna dificultas para llevar a cabo actividades de la vida diaria"; la discapacidad moderada: "Son aquellas personas que presentan una disminución o imposibilidad importante de su capacidad para realizar la mayoría de las actividades de la vida diaria, llegando incluso a requerir apoyo en labores básicas de autocuidado y superan con dificultad sólo algunas barreras del entorno". Y la discapacidad severa: "se ve reflejada en personas que ven gravemente dificultada o imposibilitada la realización de sus actividades cotidianas, requiriendo apoyo o cuidado de una 
tercera persona y no logran superar las barreras del entorno o lo hacen con gran dificultad". (ZONDEK et al., 2006, p.14 en CSASZAR, ORTEGA y RODRÍGUEZ, 2013, p. 50).

Es necesario subrayar que el Fondo Nacional de Discapacidad -FONADIS- en Chile se transformó en el Servicio Nacional de la Discapacidad -SENADIS- por mandato de la Ley $\mathrm{N}^{\circ} 20.422$ (2010) que Establece Normas sobre Igualdad de Oportunidades e Inclusión Social de Personas con Discapacidad. En su art. $\mathrm{N}^{\circ} 1$, se indica el objetivo de la mencionada LEY: "El objeto de esta ley es asegurar el derecho a la igualdad de oportunidades de las personas con discapacidad, con el fin de obtener su plena inclusión social, asegurando el disfrute de sus derechos y eliminando cualquier forma de discriminación fundada en la discapacidad".

En el Informe realizado por la Organización Mundial para la Salud en conjunto con el Banco Mundial en 2011, se señala:

En todo el mundo, las personas con discapacidad tienen peores resultados sanitarios, peores resultados académicos, una menor participación económica y unas tasas de pobreza más altas que las personas sin discapacidad. En parte, ello es consecuencia de los obstáculos que entorpecen el acceso de las personas con discapacidad a servicios que muchos de nosotros consideramos obvios, en particular la salud, la educación, el empleo, el transporte, o la información. Esas dificultades se exacerban en las comunidades menos favorecidas. Para lograr las perspectivas de desarrollo, mejores y más duraderas, que están en el corazón de los Objetivos de Desarrollo del Milenio para el 2015 y más allá, debemos emancipar a las personas que viven con alguna discapacidad y suprimir los obstáculos que les impiden participar en las comunidades, recibir una educación de calidad, encontrar un trabajo digno y lograr que sus voces sean escuchadas (OMS-BM, 2011: s/p).

Acorde con lo anterior, el Gobierno de Chile ha estado trabajando desde fines del siglo pasado en mejorar la vida de las personas discapacitadas con la legislación indicada precedentemente y con las acciones derivadas de las mismas.

Se han señalado los aspectos anteriores para agregar al período estudiado por el Dr. Caiceo, situaciones propias y políticas para la educación especial en los últimos 40 años, tanto en Chile como a nivel mundial. Según la investigación del Doctor Caiceo, la Educación Especial tiene a la fecha (2019) 167 años de historia en Chile, lo que permite hacer una distribución entre los distintos momentos, lo cual, nos lleva a una clasificación de la misma. Según los antecedentes recopilados de las investigaciones de Jaime Caiceo, es posible proponer los siguientes tres períodos entre 1852 y 1980, lapso de tiempo por él estudiado: (i) El período de surgimiento de la educación especial (1852-1926), (ii) El período del desarrollo y de los principales precursores de la educación especial (1927-1964) y (iii) Período de consolidación y de las grandes realizaciones científicas (1964-1980), los cuales serán abordados en el punto siguiente.

\section{Períodos de la Educación Especial en Chile}

\subsection{El período de surgimiento de la educación especial (1852-1926)}

El hito que se considera el inicio de la Educación Especial en Chile lo constituye la fundación de la primera escuela de sordomudos en Santiago el 27 de octubre de 1852; allí "se enseñaba gratuitamente a 'leer y escribir, dogma y moral religiosa y principios 
de gramática castellana y de aritmética' (BOLETÍN DE LEYES, 1852: Libro XVIII, núm. 10 en CAICEO, 2010a, p.35).

Cabe destacar que este acontecimiento derivó como algo normal en el desarrollo de la educación en Chile, pues en la década anterior, se habían fundado tres importantes instituciones señeras en la historia educacional del país, gracias a la existencia en el ámbito cultural de un connotado grupo de personalidades chilenas y extranjeras, acogidas por las autoridades de la época, especialmente Manuel Montt, Ministro de Justicia, Culto e Instrucción Pública en la década del cuarenta y Presidente de Chile en la siguiente: La Universidad de Chile en 1842, cuyo primer Rector fue el venezolano Andrés Bello; la Primera Escuela de Preceptores, cuyo Primer Director fue el argentino Faustino Sarmiento; y la Escuela de Artes y Oficios $^{1}$ fue el francés Pules Jaezar (CAICEO, 2009).

En 1854 se estableció, en Santiago, una segunda escuela de sordomudos, con funcionamiento gratuito. En ella se enseñaba: "... lectura, escritura, doctrina y moral cristiana, cálculo mental, costura, bordados y otros trabajos manuales adaptados a las condiciones de los alumnos y cuyo ejercicio les favorece el aprendizaje" (BOLETÍN DE LEYES, 1854, Libro XX, núm. 4 en CAICEO, 2010a, p, 35).

En 1875 se fundaron, también en Santiago, dos nuevas escuelas especiales, las cuales funcionaron en locales adecuados especialmente para ello: una escuela para la enseñanza de ciegos y otra para sordomudos (BOLETÍN DE LEYES, 1875: Libro XXII, tomo 1 en CAICEO, 2010a, p. 35).

Por otra parte, Caiceo agrega un hecho relevante, cual es la fundación de Instituto formador de Maestros para Sordomudos; al respecto señala:

en Santiago, el 10 de abril de 1889, según Decreto de ese día, en el Art. $1^{\circ}$ se indica: 'Créase el Instituto de Sordo-Mudos que tiene por objeto educar a los sordo-mudos y formar maestros para escuelas especiales que con el mismo fin sea conveniente fundar en la República'. Y en el Art. $2^{\circ}$ : 'Se adoptará exclusivamente el método de la palabra articulada y de la lectura labial. Se prohíbe el sistema mínimo y en lo posible se aplicará el ejercicio del órgano auditivo' (BOLETÍN DE LEYES, 1889: Tomo I, Primer Cuatrimestre en CAICEO, 2010a, p. 35).

A su vez, se agregó a la primera escuela de sordomudos, la Sección Ciegos, en 1900.

A la luz de los antecedentes investigados por el Dr. Jaime Caiceo en este primer período, la educación especial se centra sólo en la fundación de escuelas para sordomudos y ciegos en Santiago y se insinúa la preocupación por la deficiencia mental (CAICEO, 2010a, pp. 35-36).

\subsection{El periodo del desarrollo y de los principales precursores de la Educación Especial (1927-1964)}

En 1927 se le da importancia por primera vez a la Educación Especial, cuando en el Decreto de la Reforma Educacional, "se indica que además de las escuelas tradicionales, es necesario mantener 'escuelas-hogares para niños indigentes, débiles y de inferioridad orgánica, anormales o retrasados mentales' " (LEY 7.500, Art.17 en CAICEO, 1988a, p. 49). En este período se crearon una serie de unidades educativas en esta área:

\footnotetext{
${ }^{1}$ Esta institución se transformó en la Universidad Técnica del Estado en 1947 y en la Universidad de Santiago de Chile en 1981.
} 
* Escuelas Experimentales, sobresaliendo entre ellas la Escuela Especial de Desarrollo, creada por Decreto 5.881 de diciembre de 1928; en ella se empezaron a tratar los niños deficientes.

* Escuelas de ciegos, sordomudos y débiles mentales, según Decreto 00653 del 11 de marzo de 1929.

* Una nueva Escuela Especial de Desarrollo en Santiago, a través del Decreto 4.259 del 31 de octubre 1933; en ella se creó una sección dedicada a la educación de los niños retardados mentales, con sistema de internado. En esta escuela se destaca uno de los principales exponentes de la Educación Especial en Chile durante el siglo XX: Juan Sandoval Carrasco ${ }^{2}$, quien primero fue subdirector y luego director de la misma. Jean Cizaletti, psicólogo francés, académico de la Universidad de Chile se refiere a Juan Sandoval como quien "... trabajó mucho en lo que se llama pedagogía terapéutica, es decir, en métodos que pudieran lograr que el deficiente mental llegue al nivel que realmente pueda alcanzar desde todos los puntos de vista. Un legado que dejó, en forma indirecta, son los talleres de formación laboral" (CIZALETTI, 1886 en CAICEO, 2010a, p. 36). En esta misma Escuela Especial, por Decreto 12.289 de 1946 se creó una "clínica de conducta", efectuando en ella "una atención psicopedagógica de los niños deficientes mentales, involucrando más a la familia" (CAICEO, 2010a, p. 36). Esta escuela, se denominó en 1980, "Escuela de Educación Especial N²" y se ubicó en calle Larraín, Comuna de la Reina; su director era el profesor Heriberto Castillo.

* Por Decreto 1.336 del 21 de marzo de 1944, se creó una Escuela Especial con carácter experimental, que funcionará en la denominada Ciudad del Niño, en la Comuna de San Miguel de Santiago (CAICEO, 1988a, pp. 49-50).

Según el Dr. Jaime Caiceo, en la década del cincuenta del siglo XX, el Ministerio de Educación inició una política específica, con el fin de lograr una mayor cobertura de atención a los deficientes mentales, mejorando esa área. Esto hizo posible crear una serie de instituciones dedicadas a la educación especial en la capital:

En 1947 se fundó la Escuela Especial de Foniatría (Decreto 5.659); el 7 de diciembre de 1949, el Instituto de Pedagogía Terapéutica (Decreto 10.968) con atención a diversos trastornos (Ministerio de Educación, 1975); el 5 de junio de 1951, por Decreto Supremo 3.871, se separó la Escuela de Ciegos y Sordomudos en dos, transformándose ambas, en 1969, en Escuelas Especiales (Decreto 4.635); en 1953 se creó la Escuela de Lisiados (Decreto 7.931); el 12 de enero de 1955 se creó la Clínica Psicopedagógica (Decreto 70), para estudiar y resolver los problemas de inadaptación de alumnos de escuelas primarias; en 1957se formó un equipo mixto de especialistas, con representantes del Servicio de Neuropsiquiatría del Hospital Roberto del Río y de la Clínica Psicopedagógica, a cargo del Dr. Ricardo Olea, con el objetivo de estudiar y tratar a los niños disléxicos; el mismo año, la Clínica

\footnotetext{
${ }^{2}$ Este educador tiene una serie de publicaciones sobre su materia, entre las cuales, cabe destacar: "Orientación profesional con el niño retardado mental" en Revista de Educación, Santiago de Chile, ํ 82, pp. 38-47, 1937; El niño retardado mental, Santiago de Chile: Publicaciones Escuela Especial de Desarrollo, 1945; "Fundamentos de una política asistencial en beneficio del irregular" en Revista Avanzada $\mathrm{N}^{\circ}$ 5, Santiago de Chile, Publicación Oficial de las Escuelas Experimentales, pp. 3-9, 1960; "El curso diferencial y el niño deficiente mental leve" en El Niño Limitado, № 4, Santiago de Chile, pp. 8-17, 1969; "Reportaje a la dislexia. Primeras Jornadas Chilenas de Dislexia" en El Niño Limitado, № 11, Santiago de Chile, pp. 18-25, 1970.
} 
Psicopedagógica inició el tratamiento experimental a un grupo de disléxicos en la Escuela $\mathrm{N}^{\circ} 29$ de la capital; el 27 de julio de ese año, por Decreto 8.407, se creó un curso para sordos, anexado al Hospital Carlos Van Buren, de la ciudad de Valparaíso, con dependencia de la Escuela de Sordos de Santiago; en 1959, la Escuela Especial $N^{\circ}$ 5, en conjunto con el Hospital Arriarán, creó cursos para disléxicos con retardo mental leve; en 1961 inició sus actividades la Fundación Leopoldo Donnebaum, dirigida por él mismo, preocupada por la educación y la rehabilitación del deficiente mental (problema de oligofrenia, especialmente), constituyendo al año siguiente la Escuela de Recuperación (El Niño Limitado, 1969, p. 7); Director de ella fue Juan Sandoval, quien desde allí organizó y dirigió la revista El Niño Limitado. La fundación Donnebaum funcionaba hasta 1980 en Avenida Pedro de Valdivia, $N^{\circ}$ 156, de la capital; en 1964 se puso en marcha la Asociación Nacional Pro Niño y Adulto Deficiente Mental (CAICEO, 2010a, p. 37),

Es importante subrayar que en este período, la Educación Especial -como se ha indicado- comienza a darse en hospitales, en donde se crean escuelas para distintas necesidades especiales -como se las denomina hoy- formando equipos de profesionales (médicos, psicólogos, psiquiatras, etc.), quienes investigan experimentalmente la manera de atender de manera adecuada, no sólo a los niños con problemas físicos (sordos, mudos y ciegos), sino que también a quienes padecen deficiencia mental.

\subsection{Período de consolidación y de las grandes realizaciones científicas (1964-1980)}

Según las investigaciones del Dr. Caiceo, hay dos hechos que destacan en este período: En 1964 en la Universidad de Chile se inicia la formación sistemática de docentes en el área de la educación especial y la Reforma educacional de 1965, año en que el presidente Eduardo Frei Montalva designó la Comisión Asesora para que estudiara y propusiera soluciones a los problemas de deficiencia mental en el país, especialmente en el ámbito de protección social, educacional y económica. Ello lo concreta, a partir de un trabajo realizado por la Asociación Nacional Pro-Niño y Adulto Deficiente Mental, con lo cual "se concreta la aspiración del señor Donnebaum de contribuir a difundir los problemas de la deficiencia mental y a organizar la comunidad para conseguir dicho objetivo" (EL NIÑO LIMITADO, 1969, p. 7). Los aspectos más importantes de este período en el sector de la Educación Especial son:

* Surgimiento en varias universidades de carreras en el área y grupos de investigación entre 1964 y 1979 (Universidad de Chile, Pontificia Universidad Católica de Chile en Santiago y Sede Maule, Universidad de Concepción, Universidad Austral, Universidad Católica de Valparaíso y Universidad Católica del Norte.

* Surgimientos de carreras en el área en Institutos Profesionales y Centros de Formación Técnica a partir de 1981, año en que se crean estas modalidades de instituciones.

* Se funda el Departamento de Educación Especial en el Ministerio de Educación en 1970.

* Aprobación de Planes y Programas para las diferentes necesidades especiales, tanto transitorias como permanentes entre 1976 y 1980, cuyo detalle se verá más adelante (CAICEO, 1988b). 


\subsubsection{Preocupación por la deficiencia mental y diversificación de centros de diagnóstico y escuelas especiales}

En 1967, la Comisión antes mencionada, "después de quince meses de estudio, elaboró un programa de trabajo, que contempla diversos aspectos sobre perfeccionamiento docente, aumento de infraestructura adecuada, investigación y experimentación en el área y situación jurídica del deficiente mental" (CAICEO, 1988b, p. 136). Es digno de subrayar el proyecto de ley que preparó Enrique Silva $\mathrm{Cimma}^{3}$, con el objeto de "otorgar protección integral a este tipo de minusválido durante toda su vida, incluyendo previsión, trabajo, educación, asistencia sanitaria y asistencia legal" (ROJAS, 1967, p. 9).

En 1965, el Ministerio de Educación colocó personal especializado en psiquiatría en el Hospital Luis Calvo Mackenna, con el objeto de atender problemas de dislexia; el psicólogo Luis Bravo Valdivieso ${ }^{4}$, de renombre latinoamericano posteriormente por sus investigaciones y publicaciones en esta problemática, fue uno de los especialistas allí nombrado ${ }^{5}$, el cual permaneció en ese lugar hasta 1974; se integró al equipo multidisciplinario que se creó, la Educadora Mabel Condemarín, sobre la cual se desarrollará un punto propio más adelante. Allí mismo se creó la Escuela Especial $N^{\circ} 15$ para atender esa necesidad especial. Al año siguiente, 1966, se fundó la Escuela Especial $\mathrm{N}^{\circ}$ 19, para atender problemas de afasia y trastornos del lenguaje, incluyendo, posteriormente, la atención de niños disléxicos.

El mismo año se programó un Seminario de estudio sobre el tema, en el cual participaron los profesionales de las Escuelas Especiales $\mathrm{N}^{\circ} 15$ y $\mathrm{N}^{\circ} 19$, profundizando el estudio científico y experimental de esta anomalía. En 1967 se abrieron dos nuevas escuelas, ligadas al mismo trastorno específico y a centros hospitalarios: la escuela de Educación Especial Hospital San Borja y Escuela Especial № 2 José Joaquín Aguirre. También se creó la Escuela de Educación Especial $\mathrm{N}^{\circ} 24$ Hospital Roberto del Río, anexa al Servicio de Neuropsiquiatría del mencionado hospital. Ésta se preocupó por el tratamiento e investigación sobre la parálisis cerebral, dislexia y disfunción cerebral mínima (CAICEO, 2010a).

Por otro lado, en Viña del Mar se creó un instituto dedicado a la educación de niños con retardo mental en 1966, con la colaboración del médico neurocirujano Hugo Gárnica. Y en 1968 se fundaron dos escuelas especiales más: la Escuela Especial No 11 de Valparaíso, en el Hospital Van Buren y la Escuela Especial de Punta Arenas (CAICEO, 1988b).

El 18 de mayo de 1967 se inauguró el Centro de Experimental del Desarrollo, institución piloto destinada a la educación de niños y adolescentes deficientes mentales, dependiente de la Dirección de Educación Primaria y Normal del Ministerio de Educación, en la comuna de San Miguel, con la presencia del Presidente Frei Montalva.

En 1970 ya había en el país 44 escuelas especiales, que cubrían todos los déficits, incluido el síndrome de Down.

En el gobierno de Salvador Allende (1970-1973), habrá una preocupación especial por la capacitación laboral de los deficientes mentales. En ese contexto, la Primera Dama, Hortencia Bussi de Allende en 1972 "colocó la primera piedra del Primer Centro de Capacitación Laboral del país para adolescentes deficientes mentales, llamado Juan

\footnotetext{
${ }^{3}$ Quien fuera Contralor General de la República (1958-1964) y posteriormente, Ministro de Relaciones Exteriores (1990-1994) y Senador Designado (1998-2006).

${ }^{4}$ El Dr. Jaime Caiceo Escudero fue alumno del Dr. Luis Bravo en la Pontificia Universidad Católica de Chile, mientras estudiaba Pedagogía en Filosofía en 1966 y 1967.

${ }^{5}$ Algunas de sus publicaciones más relevantes son: Trastornos del Aprendizaje Escolar (Santiago de Chile: Teleduc, 1978), El Niño y la Escuela (Santiago de Chile: Editorial Universitaria, 1981), Dislexias y Retraso Lector (Madrid: Santillana, Aula XXI, 1985), Dislexias y Retraso Lector: Enfoque Neuropsicológico (Madrid: Santillana, 1985), Lenguaje y Dislexias: Enfoque Cognitivo del Retardo Lector (Santiago de Chile: Ediciones Universidad Católica de Chile, 1995 y México, Alfaomega, 1999), Psicología de las Dificultades de Aprendizaje (Santiago de Chile, 7a. Ed., Editorial Universitaria, 2002).
} 
Sandoval Carrasco. Éste funcionó junto a la Escuela Especial de Desarrollo (Escuela Especial 2) en la comuna de La Reina" (CAICEO, 2010a, pp. 40-41).

Antes del golpe militar de septiembre de 1973, en Chile había 8 Centros de Diagnóstico dependientes de la Universidad de Chile, el Ministerio de Educación y algunos hospitales; 34 escuelas públicas para deficientes mentales; 6 instituciones privadas sin fines de lucro en Santiago (Escuela de Recuperación Fundación Leopoldo Donnebaum, Pequeño Cottolengo Don Orione, Colegio Coocende, Instituto Apoquindo Coocela Ltda., Casa de Caridad Don Orione y Centro Psicopedagógico Holanda) y 3 escuelas privadas (Escuela Dr. Kroll, Escuela Moderna Gabriela Mistral y Colegio Juan Piaget) (CAICEO, 1988b).

\subsubsection{Formalización de planes y programas para la educación especial.}

El Dr. Jaime Caiceo, sostiene que al principio de la dictadura militar (1974) se creó, en el CPEIP, un subprograma denominado "Desarrollo de la educación diferencial". En ese contexto, el investigador de la Pontificia Universidad Católica de Chile, Luis Bravo Valdivieso, entregó el resultado de uno de sus estudios al Ministerio de Educación, denominado "El fracaso escolar básico y sus consecuencias en la educación, salud y economía", este organismo público le encargó al Centro de Perfeccionamiento, Experimentación e Investigaciones Pedagógicas -CPEIP- que organizara rápidamente un seminario sobre la educación especial en Chile. En efecto, entre el $20 \mathrm{y}$ el 23 de marzo del mismo año se concretó con participación de las diferentes entidades preocupadas por este tipo de educación: Representantes del Ministerio de Educación, de las universidades (Católica y de Chile) y del Ministerio de Salud; actuó como Presidente del evento, el destacado educador del área, Manuel Sandoval Vergara, en ese momento Jefe del Departamento de Educación Experimental y Parvularia del Ministerio de Educación.. Este Seminario funcionó en seis comisiones: Formación y perfeccionamiento docente; política de investigación; la educación básica común y la educación diferencial o especial; prevención, diagnóstico y atención precoz; centros de atención o diagnóstico y aspectos administrativos y presupuestarios; ello permitió que se abordaran las diferentes aristas de la educación diferencial con fluidez, intercambio de opiniones, clarificación de ideas y problemas, y la pronta sugerencia de acciones para superarlas; por lo mismo, en un documento del CPEIP se indica "... que las autoridades educacionales podrán recoger la determinación de una política de acción en relación a las necesidades de la Educación Especial o Diferencial que permitan un mejoramiento cualitativo y cuantitativo de esta rama de la educación (CPEIP; 1974, p. 12 en CAICEO, 2010b, p. 41).

El Prof. Caiceo señala que como una manera de buscar las mejores soluciones a los resultados del Seminario antes mencionado, en noviembre del mismo año, se realizaron distintos seminarios con directores de escuelas. Entre aquellos cabe mencionar los siguientes: "Organización escolar y realidad actual de la educación especial" y "Diagnóstico de parálisis cerebral". Se preparó, además un "Proyecto de perfeccionamiento para el personal de la educación diferencial" (CAICEO, 1988b).

Los principales resultados de la Comisión se expresaron en distintos Decretos Supremos Exentos -DSE-:

* Elaboración de planes y programas de estudio para los diferentes déficits de la educación especial: DSE $N^{\circ} 310$ de 1976 para déficit mental; DSE $\mathrm{N}^{\circ} 125$ de 1980 para déficit visual; DSE $N^{\circ} 143$ de 1980 para trastornos específicos de aprendizaje de la lecto-escritura y el cálculo; DSE N 148 de 1980 para alteraciones del lenguaje oral; DSE de 1981 para déficit auditivo; a partir de 1985 se dictan diferentes planes experimentales para Centros Laborales. 
* Creación de grupos diferenciales en las escuelas y liceos de educación regular, a fin de disminuir los índices de repitencia y deserción por Decreto $\mathrm{N}^{\circ} 457$ de 1976 para escuelas básicas, y, por Decreto $N^{\circ} 1.861$ de 1979 para los liceos, el Dr. Caiceo sostiene que

la principal característica de estos grupos diferenciales consiste en atender al alumno dentro de su medio escolar, lo que favorece el contacto entre el profesor del curso y el profesor del grupo, permitiendo hacer readecuaciones y modificaciones cuando sea necesario, a fin de dar atención complementaria al alumno que lo requiera, otorgando apoyo psicopedagógico oportuno (CAICEO, 2010a, p. 43).

* Dotación de las escuelas especiales con un gabinete técnico por Decreto $\mathrm{N}^{\circ} 911 \mathrm{de}$ 1977. Este gabinete está compuesto por un equipo de profesionales: profesor especialista, psicólogo, asistente social y fonoaudiólogo, con el objeto de "apoyar y orientar la labor de los docentes y coordinar la acción de la escuela con los padres y la comunidad" (CAICEO, 2010a, p. 43).

* Creación de organismos psicopedagógicos o Centros de Diagnóstico por Decreto $\mathrm{N}^{\circ}$ 428 de 1975. Ello permitió que los diferentes organismos públicos y privados existentes $^{6}$ entre 1981 y 1982 atendieran cerca de 25 mil escolares en todo el país, siendo el promedio de consulta anual de 300 casos en los micro-centros y de 650 en los centros (MINEDUC, 1975).

* Incentivar la creación de otras escuelas especiales: "En 1982 existían en el país 161 escuelas especiales, atendiendo a 23 mil alumnos aproximadamente. El mismo año, había 31.906 alumnos en grupos diferenciales. La ley de subvenciones de 1980 incentivó el surgimiento de escuelas especiales privadas" (MINEDUC, 1975 en CAICEO, 2010a, p. 43).

* Perfeccionamiento de profesores sin título en educación especial para atender grupos diferenciales, cursos realizados por el CPEIP y universidades.

* Dotación de plazas para docentes y profesionales no docentes.

* Entrega de material técnico y didáctico para diagnóstico y atención educativa.

* Construcción y reparación de locales escolares para la educación especial.

* Desarrollo de programas de prevención de problemas (CAICEO, 2010a, pp.42-44).

Por otro lado, a los inicios de la dictadura militar, la esposa de José Toribio Merino, miembro de la Junta Militar de Gobierno, Margarita Riofrío, fundó la Corporación del Ayuda al Niño Limitado -Coanil-, entidad de derecho privado, cooperadora de la función docente del Estado, sin fines de lucro y en 1980 ya tenía veintidós centros a lo largo de todo Chile. El objetivo de esta institución es "... propender a la atención integral del problema que presentan los sujetos con alteraciones intelectuales y laborales y/o especificas, motoras y sensoriales a fin de integrarlos eficazmente a la sociedad (COANIL, 1975 en CAICEO, 2010a, p. 44).

A finales del siglo XX, en Chile, existían varias agrupaciones de padres de niños con distintas diferencias: Unión Nacional de Padres o Amigos de Deficientes Mentales (UNPADE), la Asociación de Padres de Niños Espásticos (ASPEC) y la Asociación de Padres de Niños Autistas (ASPAU).

\footnotetext{
${ }^{6}$ En 1982 existían en el país 31 organismos pertenecientes al Ministerio de Educación, 2 de la Corporación de Ayuda al Niño Limitado -COANIL-, 2 municipales y 1 en Convenio entre Ministerio de Educación y la Universidad Austral.
} 
Paralelamente, a nivel internacional acaecieron hechos relevantes en torno a la educación especial: "Declaración de los Derechos del Retrasado Mental" en la Asamblea General 2.027 de las Naciones Unidas en 1971 y "Declaración de los Derechos de los Impedidos" en la Sesión Plenaria 2.433 de las Naciones Unidas en 1975 (CAICEO, 1988b).

Como se ha podido apreciar, efectivamente en este período hubo un avance significativo en pro de la educación especial en Chile, consolidando este tipo de educación, tan importante para atender a los niños y niñas diferentes.

\section{Aportes de Mabel Condemarín a la Educación Especial}

Mabel Condemarín Grimberg (3/11/1931-30/3/2004, por su calidad como docente chilena, destacada en el ámbito de la educación especial y discípula del Dr. Luis Bravo Valdivieso, el estado de Chile le otorgó el Premio Nacional de Ciencias de la Educación en el 2003. Su preocupación principal estuvo en los niños, especialmente con trastornos de aprendizaje, en la temática de la lecto-escritura.

Su formación es variada y profunda: Profesora Normalista en la Escuela Bongard de la Serena, 1951; Profesora de Educación en la Escuela Normal Abelardo Núñez de Santiago, 1960; estudios de Postgrado en el State College School of Educación en Los Ángeles, California, 1966-1967; Postítulo en Educación Especial en la Pontificia Universidad Católica de Chile, 1975 y Magíster en Ciencias de la Educación en la misma universidad ${ }^{7}, 1978$; Doctora en Educación por la University of Wales en Convenio con el programas Interdisciplinario de Investigaciones en Educación -PIIE- de Chile, 1990. El Dr. Caiceo dice textualmente sobre ella:

Es una educadora transgresora de su tiempo porque a pesar de su origen humilde y de padre comunista, lejana de los centros de poder, logró superarse académicamente y obtener los títulos que ella deseaba, tanto en Chile como en el extranjero. A su vez, dedicó su vida a un tema que no otorga grandes beneficios: preocuparse que los niños más pobres y con trastornos de aprendizaje aprendieran a leer y escribir compresiva y correctamente. (CAICEO, 2010b, p. 106).

En el año 1966, fue llamada a formar un equipo multidisciplinario junto al Dr. Luis Bravo, con el objeto de atender eficazmente a los niños que llegaban a Psiquiatría Infantil; fue de esta forma que empezó a darse cuenta de la importancia que tenía el poder resolver los problemas de aprendizaje de los niños especialmente en el ámbito de la lectura.

Posteriormente, el mismo Dr. Bravo la invita a participar trabajos propios del área a la Pontifica Universidad Católica de Chile en donde había creado el Postítulo en Educación Especial, integrándose como académica a esa universidad, tanto en la Facultad de Educación como en la Escuela de Psicología. Con la vuelta de la democracia, en 1990 se integra al Ministerio de Educación junto a un grupo de profesionales para participar en la Reforma Educacional que se inició ese año: Por más de una década coordinó el Programa de Mejoramiento de la Calidad y Equidad de la Educación Básica y dentro de él se encontraba el Programas de las 900 escuelas más deficitarias del país; todas sus fuerzas las puso en esos alumnos más desposeídos; en el 2002 se integró a la campaña LEM del propio Ministerio, que perseguía centrar la preocupación en la educación básica en la Lectura, Escritura y Matemática; estando en ese Programa murió de cáncer en el 2004 (CAICEO, 2010b).

El Prof. Jaime Caiceo fue compañero de ella en este Programa. 
Según el Dr. Caiceo, la preocupación de esta educadora por la lecto-escritura es muy importante (CAICEO, 2008) y su actividad pedagógica se puede sintetizar en siete ejes temáticos:

a) énfasis en el dominio profundo del lenguaje escrito, incluyendo la lectura y la literatura. b) $\mathrm{Su}$ propuesta del Enfoque o Modelo Equilibrado o balanceado en el desarrollo de la lectura inicial. c) El Programa de la Lectura Silenciosa Sostenida como complemento de todo buen plan de adquisición y adelanto en la comprensión lectora para los niveles siguientes de aprendizajes del lenguaje escrito. d) El diseño de cobertura nacional conocido como el Programa para niños con alto riesgo de fracasar en el aprendizaje y el desarrollo de la lectura y escritura. e) El Programa de Recuperación Lectora basado en la noción de medición temprana. f) Su preocupación por investigar acerca de las estrategias pedagógicas que orientasen la acción educativa con adolescentes no lectores. g) La búsqueda de fundamentación científica actualizada para sus aportes pedagógicos, por ejemplo cuando vincula el Procedimiento Cloze con el grupo de estrategias paralingüísticas, y cuando sostiene la cercanía teórica del diseño de cobertura nacional con el modelo holístico y el constructivismo. (CAICEO, 2010b, p. 114).

En cuanto a su aporte a la educación especial, según el Dr. Caiceo, se pueden sintetizar en 12 puntos; aquí se expondrán algunos de ellos:

1. Es necesario realizar un diagnóstico para descubrir tempranamente problemas en el aprendizaje de la lectura y Matemáticas, problemas de la escritura manuscrita, problemas cognitivos y síndrome de déficit atencional... 2. En el contexto del desarrollo de habilidades lectoras en los cursos intermedios, elaboró técnicas remediales para alumnos con necesidades educativas especiales basadas en la lectura oral. 3. Afirmó que el retardo mental leve (niños limítrofes), el déficit atencional con hiperkinesia, las alteraciones moderadas en la comunicación y el lenguaje y las dificultades del aprendizaje, que incluyen la dislexia y la disgrafia, son bastante frecuentes en la población escolar, y que las necesidades de aprendizaje de los niños que las posean pueden ser satisfechas, por lo menos en parte, en la situación regular del aula de clases. 4. Consideró que otro grupo de alteraciones, como el retardo mental franco, las alteraciones de visión y audición (niños ciegos y sordos), los problemas físicos invalidantes, el autismo y la psicosis, son problemas severos pero poco frecuentes. Sostuvo que satisfacer las necesidades específicas de los niños que las padecen implica el tratamiento con fármacos, el trabajo con la familia, la psicoterapia individual o grupal y la enseñanza correctiva o remedial. 5. En relación con los niños sordos, indicó la importancia de determinar las posibilidades del Procedimiento Cloze como medio de diagnóstico y desarrollo, ya que una gran parte del aprendizaje de los alumnos nooyentes se logra por medio de la lectura silenciosa... 6. Efectuó una caracterización de los niños limítrofes, de los niños con déficit atencional con hiperkinesia, de los niños con alteraciones en la comunicación y el lenguaje $\mathrm{y}$ de los niños con dificultades de 
aprendizaje, proporcionando sugerencias para satisfacer sus específicas necesidades educativas. En relación a los niños limítrofes, propuso que los principales indicadores del problema son un lento ritmo de aprendizaje, atraso consistente en la mayoría de las áreas de desarrollo y problemas adaptativos... (CAICEO, 2011, pp. 183-184).

Esta educadora es vastamente conocida en Latinoamérica y el artículo del Dr. Caiceo citado precedentemente sobre ella, es consultado en los tres últimos sobre 10 veces a la semana; tiene, a su vez, muchas publicaciones sobre las áreas de Aprendizaje, Evaluación y Educación; sobre Lecto-Escritura y sobre Educación Especial; algunas de ellas del último grupo son: Condemarín, Mabel "Niños con necesidades educativas especiales" en Dr. Winter, E.A. \& Dr. Puentes, R.R. Medicina Infantil (pp. 1093-1099), Santiago de Chile: Speed Printer, 1991; Condemarín, Mabel; Blomquist, Marlys La dislexia (16a. ed.), Santiago de Chile: Ed. Universitaria, 1998; Condemarín, Mabel; Milicic, Neva Test de Cloze (3a. ed.), Santiago de Chile: Ed. Andrés Bello, 2004; Condemarín, Mabel; Gorostegui, María Elena; Milicic, Neva Déficit atencional (3a. ed.), Santiago de Chile: Ed. Planeta Chilena, 2004.

\section{Conclusiones}

Como se ha podido apreciar, el tema de la inclusión en el Chile republicano es de larga data: mitad del siglo XIX en adelante y el Dr. Jaime Caiceo, lo ha resaltado bastante (Caiceo, 2005) cumpliendo los objetivos propuestos. Se puede destacar que, en el campo de la Educación Especial, Chile es pionero en Sudamérica, al crearse en 1852, la primera Escuela de Sordomudos. Además, el mayor desarrollo de la Educación Especial en Chile, en el último período, se debió al interés despertado por ella en las universidades, en las cuales no solo se comienza a formar profesionales desde 1964, sino que sobre todo, se investiga y se publica sobre el tema.

Además, es relevante que en el último período descrito se diversificó en la preocupación, el estudio y la atención de los diferentes déficits, se tomó conciencia como una educación indispensable en el sistema educacional del país, los padres asumieron de que no hay que avergonzarse de que los hijos nazcan con algún tipo de déficit sino que es necesario tratarlos con los especialistas apropiados. Se experimentó con éxito que el trabajo educacional produce mejores resultados con un equipo multidisciplinario. Recalcar, además, la cantidad de investigaciones y aportes teórico-prácticos a la Educación Especial, subrayando la valorización a las personas con déficit como seres humanos con los mismos derechos que los normales y que, en su nivel, también se perfeccionan y progresan. Se han destacado, a su vez, a varios educadores chilenos, conocidos en toda América, especialmente el Prof. Juan Sandoval Carrasco, el Dr. Luis Bravo Valdivieso y la Dra. Mabel Condemarín Grimberg.

Actualmente existen reformas que han ido fomentándose con políticas públicas, pero por tratarse este trabajo de un período determinado, será necesario continuarlo en una nueva investigación, donde se abordará los tiempos actuales. Sin embargo, es necesario señalar que la Ley de Inclusión que entró en vigencia en el 2016 sostiene que todos los niños, con dificultades o sin ellas, deben estar en la escuela común; ello ha significado que en las escuelas se han creado problemas graves, pues las personas con necesidades especiales permanentes (sordo-mudos, síndrome de Down, deficiencia mental severa, etc.) es mejor tratarlos en una Escuela Especial (CAICEO, 2018). 


\section{Referencias}

BIBLIOTECA DEL CONGRESO NACIONAL. Boletín de Leyes, Libro XVIII, núm. 10. Santiago de Chile, 1852.

BIBLIOTECA DEL CONGRESO NACIONAL. Boletín de Leyes, Libro XX, núm. 4. Santiago de Chile, 1854.

BIBLIOTECA DEL CONGRESO NACIONAL. Boletín de Leyes, Libro XXII, tomo 1. Santiago de Chile, 1875.

BIBLIOTECA DEL CONGRESO NACIONAL. Boletín de Leyes, Tomo I, Primer Cuatrimestre. Santiago de Chile, 1889.

BIBLIOTECA DEL CONGRESO NACIONAL. Ley $N^{\circ}$ 19.284: Establece normas para la plena integración social de personas con discapacidad. Santiago de Chile. Promulgada en el Diario Oficial 14 de enero de 1994.

BIBLIOTECA DEL CONGRESO NACIONAL. Ley $N^{\circ}$ 20.422: Establece normas sobre igualdad de oportunidades e inclusión social de personas con discapacidad. Santiago de Chile: Promulgada en el Diario Oficial el 10 de febrero de 2010.

BIBLIOTECA DEL CONGRESO NACIONAL. Ley $N^{\circ}$ 20.845: De inclusión escolar que regula la admisión de los y las estudiantes, elimina el financiamiento compartido y prohíbe el lucro en establecimientos educacionales que reciben aportes del estado. Santiago de Chile: Promulgada en el Diario Oficial el 8 de junio de 2015.

CAICEO, Jaime. La Educación Especial en Chile: Un Esbozo de su Historia. Revista de Pedagogía, $\mathrm{N}^{\circ} 306$, pp. 47-51, 1988a.

CAICEO, Jaime. La Educación Especial en Chile: Período de Consolidación. Revista de Pedagogía, n.309, pp. 135-141, 1988 b.

CAICEO, Jaime. Procesos de Inclusión y Exclusión en el Sistema Educativo Chileno durante los Siglos XIX y XX. Perspectivas Educacionales, $N^{\circ}$ 5, Universidad Metropolitana de Ciencias de la Educación, pp. 75-82, 2005.

CAICEO, Jaime. "Mabel Condemarín: Lecto Escritura y Educación de Calidad". Revista Intramuros, Año 8, N 22, Universidad Metropolitana de Ciencias de la Educación, pp. 31-38, 2008.

CAICEO, Jaime. Estado, Iglesia y Sistema Educativo durante la República en Chile. Revista HISTEDBR On-line, n.35, Universidade Estadual de Campinas, pp. 3-18, setembro, 2009. https://doi.org/10.20396/rho.v9i35.8639610

CAICEO, Jaime. Esbozo de la Educación Especial en Chile: 1850-1980. Revista Educación y Pedagogía, Vol. 22, n.57, p. 31-49, 2010a.

CAICEO, Jaime. Amanda Labarca, Irma Salas y Mabel Condemarín: Tres Educadoras Laicas y Feministas del siglo XX en Chile. Revista Teoria e Prática da Educaçāo, V. 13, $\mathrm{N}^{\circ}$ 1, Universidade Estadual de Maringá, PR, pp. 105-116, setembro, $2010 \mathrm{~b}$. 
CAICEO, Jaime. Mabel Condemarín: Una Educadora Diferencial Chilena. Revista Educación y Humanismo, Vol. 13, $\mathrm{N}^{\circ}$ 21, pp. 174-190, 2011.

CAICEO, Jaime. Entrevista, realizada en Santiago de Chile por Estela Socías Muñoz el 15 de noviembre de 2018 .

CENTRO DE PERFECCIONAMIENTO, EXPERIMENTACIÓN E INVESTIGACIONES PEDAGÓGICAS -CPEIP-. Seminario de Educación Especial o Diferencial. Lo Barnechea: MINEDUC-CPEIP, 1974.

CSASZAR, Shani; ORTEGA, Jocelyn; RODRÍGUEZ, María José. Políticas Educativas y Educación Especial en Chile (1990 A 2010). Santiago de Chile: Tesis para Optar al Título de Profesora de Educación Diferencial y al Grado de Licenciada en Educación, Universidad Academia de Humanismo Cristiano, 2013.

MINISTERIO DE EDUCACIÓN DE CHILE. Antecedentes sobre la Educación Diferencial en Chile. Santiago de Chile: Centro de Perfeccionamiento, Experimentación e Investigaciones Pedagógicas -CPEIP-, 1975.

ORGANIZACIÓN MUNDIAL DE LA SALUD; BANCO MUNDIAL. Informe Mundial sobre la Discapacidad. Ginebra: OMS; BM, en https://www.who.int/disabilities/ world_report/2011/summary_es.pdf. Consultada el 15 de mayo de 2011.

ROJAS, Patricio. El Problema de la Deficiencia Mental en Chile. Revista de Educación, $\mathrm{N}^{\circ} 1$, Santiago de Chile, pp. 5-18, 1967. 